Pure and Applied Mathematics Quarterly

Volume 8, Number 2

(Special Issue: In honor of

F. Thomas Farrell and Lowell E. Jones, Part 2 of 2)

$479-496,2012$

\title{
Remarks On and Around Bounded Differential Forms
}

\author{
Anna Wienhard
}

\begin{abstract}
Various exotic cohomology groups can be defined by imposing boundedness conditions on cochains. This note discusses what happens if we impose boundedness conditions in the standard deRham complex of differential forms on a Riemannian manifold and surveys relations of the resulting bounded deRham cohomology to bounded cohomology and $l_{\infty}$-cohomology. Keywords: Bounded Differential Forms, Symmetric Spaces, Bounded Cohomology, $l_{\infty}$-cohomology.
\end{abstract}

\section{INTRODUCTION}

Given an exact smooth differential form $\alpha$ on a Riemannian manifold $M$ there exists a primitive, that is a smooth differential form $\beta$ such that $d \beta=\alpha$. Sometimes it is useful to consider the growth of $\beta$ and in particular to address the question when a differential form $\alpha$ admits a bounded primitive.

This question has been raised by Gromov in [14] and [13] when $\alpha$ is of degree 2 and in [12] when $\alpha$ is the volume form. In degree 2 the existence of a bounded primitive is strongly related to co-filling inequalities and hyperbolicity phenomena. When $M=\widetilde{X}$ is the universal cover of a negatively curved Riemannian manifold $X$, every closed form $\alpha$ of degree $\geq 2$ on $M$ admits a bounded primitive. When $X$ is a Kähler manifold with Kähler form $\omega$ Gromov gave the following definition in [13] : $X$ is called Kähler hyperbolic if the pull-back $\widetilde{\omega}$ of $\omega$

Received October 12, 2007.

The author was partially supported by the National Science Foundation under agreement No. DMS-0604665. 
to the universal covering $\widetilde{X}$ admits a bounded primitive *. Furthermore he proved that for closed Kähler hyperbolic manifolds the space $\mathcal{H}^{p, q}(\widetilde{X})$ of $L^{2}$-harmonic forms of type $(p, q)$ vanishes except for $p+q=\operatorname{dim}_{\mathbf{C}}(X)$. This implies in particular that Kähler hyperbolic manifolds satisfy that $\operatorname{sign}(\chi(X))=(-1)^{\operatorname{dim}_{\mathbf{C}} X}$, which is also known as "Hopf's conjecture". Gromov suggests that Kähler hyperbolic manifolds should be regarded as being hyperbolic with respect to their complex geometry. This indeed seems appropriate when considering some Kähler hyperbolic manifolds which are not negatively curved, as for example compact quotients of Hermitian symmetric spaces or the moduli space of Riemann surfaces of genus $g \geq 2$ which admits several Kähler metrics with respect to which it is Kähler hyperbolic [17]. Both these examples are known to exhibit some hyperbolicity phenomena.

In this note we consider the problem of finding bounded primitives in all degrees and relate it to vanishing (and non vanishing) of various cohomology groups. In particular, for symmetric spaces of noncompact type we show that in several cases bounded primitives exist.

Proposition 1. Let $M$ be a symmetric space of noncompact type of rank $r_{M}$ and $\alpha$ a closed bounded smooth differential form of degree $k$ on $M$. If $k \geq r_{M}+1$ then there exists a bounded $(k-1)$-form $\beta$ on $M$ with $d \beta=\alpha$.

When we restrict to differential forms invariant under the group of isometries we can drop the condition on the degree of $\alpha$.

Proposition 2. Let $M$ be a symmetric space of noncompact type and $G=$ $\operatorname{Isom}(M)^{\circ}$ the connected component of the group of isometries. Let $\alpha$ be a $G$ invariant differential form on $M$, then there exists a bounded differential form $\beta$ with $d \beta=\alpha$. Moreover, $\beta$ can be chosen to be P-invariant for a minimal parabolic subgroup $P<G$.

Corollary 3. Let $G$ be a semisimple Lie group with finite center and $P<G$ a minimal parabolic subgroup. Then the restriction map $\mathrm{H}_{\mathbf{c}}^{\bullet}(G, \mathbf{R}) \rightarrow \mathrm{H}_{\mathbf{c}}^{\bullet}(P, \mathbf{R})$ in continuous cohomology is zero.

*A variant of this notion, namely symplectically hyperbolic manifolds has been introduced and studied by Polterovich in [20], see also [15]. 
Remark 4. The proofs rely on a Poincaré-Lemma with respect to a point at infinity. Proposition 1 has also been proven in [8]. Proposition 2 might be known to experts, but the author is not aware of any reference.

Let us consider the space

$$
\Omega_{\infty}^{k}(M):=\left\{\alpha \in \Omega^{k}(M) \mid\|\alpha\|_{\infty}<\infty,\|d \alpha\|_{\infty}<\infty\right\}
$$

of bounded differential forms on $M$ whose differential is also bounded, and the corresponding subcomplex of the standard deRham complex. We denote by $\mathrm{H}_{\mathrm{bdR}}^{\bullet}(M, \mathbf{R})=\mathrm{H}\left(\Omega_{\infty}^{\bullet}(M)\right)$ the cohomology of this subcomplex and call it the bounded deRham cohomology of $M$. Proposition 1 translates to a vanishing result for $\mathrm{H}_{\mathrm{bdR}}^{\bullet}(M, \mathbf{R})$ above the rank.

When $\widetilde{X}$ is a Hermitian symmetric space the form $\widetilde{\omega}$ is not only the coboundary of a bounded one-form, but moreover the complex area (measured with respect to $\widetilde{\omega})$ of geodesic triangles in $\widetilde{X}$ is uniformly bounded. As a consequence $\widetilde{\omega}$ defines a bounded cohomology class. This stronger property (together with further knowledge) has been applied extensively to study homomorphisms into the isometry group of $\tilde{X}$, see $[2,4,5,6]$. The example of Hermitian symmetric spaces motivates to investigate whether there is a more general relation between the existence of bounded primitives and whether the differential form defines a bounded cohomology class. When $G$ is a connected semisimple Lie group with finite center, $M$ its associated symmetric space, and $L<G$ a closed subgroup, then any continuous cohomology class $\kappa \in \mathrm{H}_{\mathrm{c}}^{k}(L, \mathbf{R})$ can be realized by an $L$-invariant differential form on $M$. Recently Burger and Iozzi showed in [3] that the comparison map $c_{L}^{\bullet}: \mathrm{H}_{\mathrm{cb}}^{\bullet}(L, \mathbf{R}) \rightarrow \mathrm{H}_{\mathrm{c}}^{\bullet}(L, \mathbf{R})$ (see Section 3.1 for the definition of the continuous bounded cohomology $\mathrm{H}_{\mathrm{cb}}^{\bullet}(G, \mathbf{R})$ of a group $\left.G\right)$ factors as

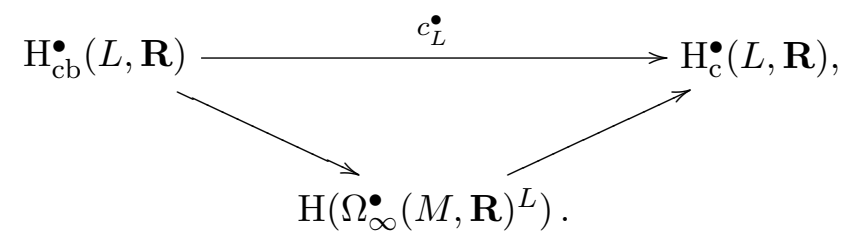

In particular, a continuous cohomology class $\kappa \in \mathrm{H}_{\mathrm{c}}^{k}(L, \mathbf{R})$ which lies in the image of $c_{L}^{(k)}$ can be represented by a bounded $L$-invariant differential form. 
More importantly in our context is the following statement which is a direct consequence of the more precise result proven in [3, Lemma 3.3], which we will recall in Section 3.

Proposition 5. Let $\kappa \in \mathrm{H}_{\mathrm{c}}^{k}(L, \mathbf{R})$. Assume that $\kappa$ lies in the image of $c_{L}^{(k)}$, then $\kappa$ can be represented by a bounded $L$-invariant differential form $\alpha$ on $M$ which admits a bounded primitive $\beta \in \Omega_{\infty}^{k-1}(M, \mathbf{R})$.

It is a very interesting open question whether the comparison map $c_{G}^{\bullet}$ : $\mathrm{H}_{\mathrm{cb}}^{\bullet}(G, \mathbf{R}) \rightarrow \mathrm{H}_{\mathrm{c}}^{\bullet}(G, \mathbf{R})$ is surjective (for $G$ as above). From this point of view Proposition 2 states that the necessary condition given by Proposition 5 is satisfied. Similarly Proposition 1 shows that, when $\Gamma<G$ is a cocompact lattice, the corresponding necessary condition for the surjectivity of $c_{\Gamma}^{\bullet}: \mathrm{H}_{\mathrm{cb}}^{\bullet}(\Gamma, \mathbf{R}) \rightarrow$ $\mathrm{H}_{\mathrm{c}}^{\bullet}(\Gamma, \mathbf{R})$ in degrees $\bullet \geq r_{M}+1$ is satisfied. Unfortunately, this does not even come close to any sufficient condition.

When $\Gamma$ is the fundamental group of an aspherical manifold $X$, the bounded deRham cohomology of the universal cover $\widetilde{X}$ is strongly related to another exotic cohomology, the $l_{\infty}$-cohomology (or bounded valued cohomology) $\mathrm{H}_{\infty}^{\bullet}(\Gamma, \mathbf{R})$, which has been introduced and studied by Gersten in $[9,10,11]$ for finitely generated groups. More precisely one has

Proposition 6. [11] The $l_{\infty}$-cohomology $\mathrm{H}_{\infty}^{k}(\Gamma, \mathbf{R})$ is isomorphic to a direct summand of $\mathrm{H}_{\mathrm{bdR}}^{k}(\tilde{X}, \mathbf{R})$.

Bounded cohomology, (continuous) cohomology and $l_{\infty}$-cohomology are connected by a sequence of natural maps

$$
\mathrm{H}_{\mathrm{b}}^{\bullet}(\Gamma, \mathbf{R}) \stackrel{c_{\Gamma}^{\bullet}}{\longrightarrow} \mathrm{H}^{\bullet}(\Gamma, \mathbf{R}) \stackrel{h_{\Gamma}^{\bullet}}{\longrightarrow} \mathrm{H}_{\infty}^{\bullet}(\Gamma, \mathbf{R})
$$

whose composition is zero. The behavior of the natural maps $c_{\Gamma}^{\bullet}: \mathrm{H}_{\mathrm{cb}}^{\bullet}(\Gamma, \mathbf{R}) \rightarrow$ $\mathrm{H}_{\mathrm{c}}^{\bullet}(\Gamma, \mathbf{R})$ and $h_{\Gamma}^{\bullet}: \mathrm{H}(\Gamma, \mathbf{R}) \rightarrow \mathrm{H}_{\infty}(\Gamma, \mathbf{R})$ in general is quite mysterious, but in the cases where it is known, their properties are complementary to each other:

- In degree 1 the map $h_{\Gamma}^{(1)}$ is injective, whereas the image of $c_{\Gamma}^{(1)}$ is zero because $\mathrm{H}_{\mathrm{cb}}^{1}(\Gamma, \mathbf{R})=0$.

- If $\Gamma$ is amenable $h_{\Gamma}^{\bullet}$ is injective [9, Theorem 10.13], whereas $\mathrm{H}_{\mathrm{cb}}^{\bullet}(\Gamma, \mathbf{R})=0$ for $\bullet \geq 1$. 
- If $\Gamma$ is hyperbolic $h_{\Gamma}^{\bullet}$ is the zero map in degrees $\bullet \geq 2$ [11, Corollary 4.4], whereas $c_{\Gamma}^{\bullet}$ is surjective in degrees $\bullet \geq 2[18]$.

Proposition 1 together with Proposition 6 implies

Proposition 7. Let $G$ be a connected semisimple Lie group with finite center, $M$ its associated symmetric space, and $\Gamma<G$ a cocompact lattice, then

$$
h_{\Gamma}^{\bullet}: \mathrm{H}^{\bullet}(\Gamma, \mathbf{R}) \rightarrow \mathrm{H}_{\infty}^{\bullet}(\Gamma, \mathbf{R})
$$

is the zero map in degrees $\bullet \geq r_{M}+1$.

It is tempting to ask the following

Question 8. When is the sequence of natural maps in (1) exact?

It seems unreasonable to expect a simple answer to this question. For cocompact lattices $\Gamma<G$ exactness in degrees $\bullet \geq r_{M}+1$ would imply the surjectivity of the comparison map $c_{\Gamma}^{\bullet}$ in the corresponding degrees. The surjectivity of the comparison map is a very difficult problem, e.g. the surjectivity of $c_{\Gamma}^{(\operatorname{dim}(M))}$ is equivalent to the positivity of the simplicial volume of $M / \Gamma$. In full generality the positivity of the simplicial volume has only recently been proven by Lafont and Schmidt [16], and by Bucher [1] in the case when $G$ is locally isomorphic to $\operatorname{SL}(3, \mathbf{R})$, which is excluded in [16].

In Section 5 we introduce a variant of Gersten's $l_{\infty}$-cohomology which can be defined more generally for second countable topological groups. It allows to address the above mentioned questions in this more general context. We show that when $\Gamma$ is a finitely generated group admitting a $K(\Gamma, 1)$-space with finite $n$ skeleton, then this variant agrees with Gersten's $l_{\infty}$-cohomology in degrees $i \leq n$.

The paper is organized as follows: In Section 2 we prove Proposition 1 and Proposition 2. We review bounded cohomology and the construction of Burger and Iozzi in Section 3. Section 4 and Section 5 review and discuss Gersten's $l_{\infty}$-cohomology and our variant of bounded valued cohomology.

\section{Bounded Differential Forms on Symmetric Spaces}

\subsection{Bounded deRham cohomology. Let}

$$
\Omega_{\infty}^{k}(M):=\left\{\alpha \in \Omega^{k}(M) \mid\|\alpha\|_{\infty}<\infty,\|d \alpha\|_{\infty}<\infty\right\},
$$


be the space of bounded differential forms on $M$ whose differential is also bounded, where

$$
\|\alpha\|_{\infty}=\sup _{m \in M} \sup _{v_{1}, \ldots, v_{k} \in T_{m}^{1} M}\left|\alpha_{m}\left(v_{1}, \ldots, v_{k}\right)\right|
$$

and $T_{m}^{1} M$ denotes the unit tangent sphere of $M$ in $m \in M$. By definition the exterior differential satisfies $d\left(\Omega_{\infty}^{k}(M)\right) \subset \Omega_{\infty}^{k+1}(M)$, so $\left(\Omega_{\infty}^{k}(M), d\right)$ is a subcomplex of the standard deRham complex. The cohomology of this subcomplex is called bounded deRham cohomology and is denoted by

$$
\mathrm{H}_{\mathrm{bdR}}^{\bullet}(M, \mathbf{R}) \text {. }
$$

When $N$ is a compact Riemannian manifold and $M=\widetilde{N}$ its universal cover, then we have a natural map

$$
\mathrm{H}_{\mathrm{dR}}^{\bullet}(N, \mathbf{R}) \cong \mathrm{H}_{\mathrm{bdR}}^{\bullet}(N, \mathbf{R}) \rightarrow \mathrm{H}_{\mathrm{bdR}}^{\bullet}(M, \mathbf{R}) .
$$

2.2. Vanishing Results for Bounded deRham cohomology. Let $X$ be a symmetric space of noncompact type and $G=\operatorname{Isom}(X)^{\circ}$ the connected component of its isometry group. Let $d_{X}$ be a $G$-invariant Riemannian distance function on $X$. The rank of $X$, denoted by $r_{X}$, is the maximal dimension of an isometrically and totally geodesically embedded Euclidean subspace in $X$. Such a Euclidean subspace of maximal dimension is called a maximal flat in $X$. The group $G$ acts transitively on the set of maximal flats.

The visual boundary $\partial X$ of $X$ is defined as

$$
\partial X:=\left\{c: \mathbf{R}^{+} \rightarrow X \mid c \text { is a unit speed geodesic ray }\right\} / \sim,
$$

where $c \sim c^{\prime}$ if and only if $c$ and $c^{\prime}$ are asymptotic, that is $\sup _{t} d_{X}\left(c(t), c^{\prime}(t)\right)<\infty$. Given a base point $x_{0} \in X$ we can identify $\partial X$ with the unit tangent sphere $T_{x_{0}}^{1} X$ and we endow $\partial X$ with the topology of the latter.

A geodesic ray $c$ is called regular if the geodesic $\bar{c}$ extending $c$ is contained in a unique maximal flat. A point $\xi \in \partial X$ is said to be regular if $\xi$ can be represented by a regular geodesic ray. Then every geodesic ray representing $\xi$ is regular. The stabilizer $P=\operatorname{Stab}_{G}(\xi)$ of a regular point $\xi \in \partial X$ is a minimal parabolic subgroup in $G$.

Let $\xi \in \partial X$ be a regular point and $c: \mathbf{R} \rightarrow X$ a regular geodesic such that $c(0)=x_{0}$ and $\left.c\right|_{\mathbf{R}^{+}}$represents $\xi$. The Busemann function $B_{\xi}: X \times X \rightarrow \mathbf{R}$, 
defined by

$$
B_{\xi}(x, y):=\lim _{t \rightarrow \infty}\left(d_{X}(x, c(t))-d_{X}(y, c(t))\right),
$$

measures the relative distance from $\xi$. We set $B_{\xi}^{0}(x):=B_{\xi}\left(x, x_{0}\right)$ and call it the normalized Busemann function. Then $B_{\xi}^{0}(c(0))=0$ and $B_{\xi}^{0}(c(t))=-t$. The level sets of $B_{\xi}^{0}$ are horospheres centered at $\xi$. Let $V_{\xi}=-\operatorname{grad} B_{\xi}^{0}$ be the negative gradient vector field of $B_{\xi}^{0}$ and $\phi_{t}^{\xi}: X \rightarrow X$ the corresponding flow. Then $\left|V_{\xi}\right|=1, V_{\xi}$ and $\phi_{t}^{\xi}$ are independent of the choice of the geodesic $c$ representing $\xi \in \partial X$ and of the normalization. In particular $V_{\xi}$ and $\phi_{t}^{\xi}$ are $\operatorname{Stab}_{G}(\xi)$-invariant.

Proposition 2.1. Let $\alpha \in \Omega^{k}(X)$ be a closed bounded smooth differential $k$-form on $X$ such that for every maximal flat $F \subset X$ and every $x \in F$

$$
\alpha_{\left.\right|_{T_{x} F}}=0
$$

Then there exists $\beta \in \Omega^{k-1}(X)$ such that

$$
d \beta=\alpha \text { and } \beta \text { is bounded. }
$$

Proof. The basic idea is to prove a Poincaré Lemma with respect to a point at infinity. For this we replace the distance function from a point by the Busemann function with respect to a regular point $\xi \in \partial X$. The condition (2) will ensure that the improper integral we have to consider is well defined.

Let $\xi \in \partial X$ be a regular point. For every $x \in X$ consider $c_{x}: \mathbf{R} \rightarrow X$ defined by $c_{x}(t):=\phi_{t}^{\xi}(x)$. Then $c_{x}$ is the unique unit speed geodesic through $x=c_{x}(0)$ which is asymptotic to $\xi$ at $+\infty$. In particular $c_{x}$ is a regular geodesic and is hence contained in a unique maximal flat $F_{x}$. The family of flats $F_{x}, x \in X$ constitute a smooth foliation $\mathcal{F}$ of $X$ by totally geodesic Euclidean subspaces.

For any unit tangent vector $u \in T X$ perpendicular to $\mathcal{F}$ the sectional curvature $K\left(u, V_{\xi}\right)$ is negative. By homogeneity of $X$ the sectional curvature $K\left(u, V_{\xi}\right)$ is bounded away from 0 by a uniform constant independent of $u$. In particular, the Jacobi fields of geodesic variations by geodesics $c_{x}$ and perpendicular to $\mathcal{F}$ decay uniformly exponentially. Since $\alpha$ vanishes along $\mathcal{F}$ by assumption, there exists $\kappa>0$, which does not depend on $\alpha$ such that for all $v_{1}, \ldots v_{k} \in T_{x}^{1} X$

$$
\left|\left(\phi_{t}^{\xi}\right)^{*} \alpha\left(v_{1}, \ldots, v_{k}\right)\right| \leq e^{-t \kappa}\|\alpha\|_{\infty} .
$$


In particular the integral

$$
\alpha^{\prime}:=-\int_{0}^{\infty}\left(\phi_{t}^{\xi}\right)^{*} \alpha d t
$$

is well defined, and $\alpha^{\prime}$ is a bounded smooth differential form on $X$. By construction $\mathfrak{L}_{V_{\xi}} \alpha^{\prime}=\alpha$ and $d \alpha^{\prime}=0$, where $\mathfrak{L}_{V_{\xi}}$ denotes the Lie derivative in the direction of $V_{\xi}$.

Setting

$$
\beta=i_{V_{\xi}} \alpha^{\prime}
$$

where $i_{V_{\xi}}$ denotes the contraction with the vector field $V_{\xi}$, we get that

$$
\alpha=\mathfrak{L}_{V_{\xi}} \alpha^{\prime}=d\left(i_{V_{\xi}} \alpha^{\prime}\right)+i_{V_{\xi}}\left(d \alpha^{\prime}\right)=d \beta .
$$

Since $\alpha^{\prime}$ is bounded and $\left|V_{\xi}\right|=1$, we have that $\beta$ is bounded.

The dimension of a maximal flat $F \subset X$ is $r_{X}$, so the condition that $\alpha_{\left.\right|_{X X} F}=0$ for every maximal flat $F \subset X$ and every $x \in F$ is satisfied if $\alpha \in \Omega^{k}(X)$ with $k>r_{X}$.

Corollary 2.2. Let $\alpha \in \Omega_{\infty}^{k}(X)$ be a closed form, $k \geq r_{X}+1$, then $[\alpha]=0 \in$ $\mathrm{H}_{\mathrm{bdR}}^{k}(X, \mathbf{R})$. In particular $\mathrm{H}_{\mathrm{bdR}}^{k}(X, \mathbf{R})=0$ for $k \geq r_{X}+1$.

Another instance when the vanishing condition (2) is satisfied is when $\alpha \in$ $\Omega^{k}(X)$ is a $G$-invariant form.

Lemma 2.3. Let $\alpha \in \Omega^{k}(X)^{G}$, then $\alpha_{\left.\right|_{T_{x}}}=0$ for every maximal flat $F \subset X$ and every $x \in F$.

Proof. Since $\alpha$ is $G$-invariant and $G$ acts transitively on the set of (pointed) maximal flats we just have to prove that there exists some $x_{0} \in X$ and a maximal flat $F_{0}$ through $x_{0}$ such that $\alpha_{\left.\right|_{T_{0} F_{0}}}=0$.

Let $x_{0} \in X, K=\operatorname{Stab}_{G}\left(x_{0}\right)$ a maximal compact subgroup of $G$ and $\mathfrak{g}=\mathfrak{k} \oplus \mathfrak{p}$ the Cartan decomposition of the Lie algebra $\mathfrak{g}$ of $G$ with respect to $x_{0}$. Then $T_{x_{0}} X$ can be identified with $\mathfrak{p}$. Let $\mathfrak{a} \subset \mathfrak{p}$ be a maximal abelian subalgebra contained in $\mathfrak{p}$ and $A=\exp (\mathfrak{a})<G$ the corresponding subgroup of $G$. Then $F_{0}:=A x_{0}$ is a maximal flat through $x_{0}$ and $T_{x_{0}} F_{0}$ is naturally identified with $\mathfrak{a}$. The maximal compact group $K$ acts on $T_{x_{0}} X \simeq \mathfrak{p}$ via the isotropy representation. This induces an action of the Weyl group $W(A)=N_{K}(A) / Z_{K}(A)$ of $A$ on $\mathfrak{a}$. Here $N_{K}(A)$ and 
$Z_{K}(A)$ are the normalizer and centralizer of $A$ in $K$ respectively. The restriction of the $G$-invariant form $\alpha$ to $\mathfrak{a}$ is a $W(A)$-invariant skew-symmetric multi-linear form on $\mathfrak{a}$.

Let $\Delta=\left\{\alpha_{1}, \cdots \alpha_{r_{X}}\right\} \subset \mathfrak{a}^{*}$ be a system of positive simple roots of $\mathfrak{g}$ relative to $\mathfrak{a}$. Let $\left\{\alpha_{1}^{*}, \cdots \alpha_{r_{X}}^{*}\right\}$ be the basis of $\mathfrak{a}$ dual to $\left\{\alpha_{1}, \cdots \alpha_{r_{X}}\right\} \subset \mathfrak{a}^{*}$. The Weyl group $W(A)$ acts on $\mathfrak{a}$ by reflections in the hyperplanes $H_{\alpha_{i}}=\operatorname{ker} \alpha_{i}$. We can choose $g_{\alpha_{i}} \in N_{K}(A)$ such that $g_{\alpha_{i}}$ generates the reflection in $H_{\alpha_{i}}$. Then $\alpha_{\left.\right|_{\mathfrak{a}}}$ is invariant by $g_{\alpha_{i}}$. But since the vectors $\alpha_{i}^{*}$ are perpendicular to $H_{\alpha_{i}}$ and span $\mathfrak{a}$ this implies $\alpha_{\left.\right|_{T_{0} F_{0}}}=\left.\alpha\right|_{\mathfrak{a}}=0$.

Corollary 2.4. Let $\alpha \in \Omega^{k}(X)^{G}$, then there exists $\beta \in \Omega_{\infty}^{k-1}(X)$ such that $d \beta=\alpha$. Moreover, $\beta$ can be constructed such that $\beta \in \Omega^{k-1}(X)^{P}$.

Proof. Note first that since $\alpha$ is $G$-invariant we have in particular that $\alpha$ is closed and bounded. By Lemma 2.3 and Proposition 2.1 there exists $\beta \in \Omega_{\infty}^{k-1}(X)$ such that $d \beta=\alpha$.

Since $\alpha$ is $G$-invariant, it is in particular $P$-invariant for $P=\operatorname{Stab}_{G}(\xi)$ with $\xi \in \partial X$ being a regular point. Furthermore $V_{\xi}$ and $\phi_{t}^{\xi}$ are $P$-invariant, so the differential form

$$
\beta=i_{V_{\xi}}\left(-\int_{0}^{\infty}\left(\phi_{t}^{\xi}\right)^{*} \alpha d t\right)
$$

constructed above is a $P$-invariant differential form.

Corollary 2.5. The restriction map $\mathrm{H}_{\mathrm{c}}^{\bullet}(G, \mathbf{R}) \rightarrow \mathrm{H}_{\mathrm{c}}^{\bullet}(P, \mathbf{R})$ is zero.

Proof. For every closed subgroup $L<G$ in a connected semisimple Lie group with finite center, the continuous cohomology $\mathrm{H}_{\mathbf{c}}^{\bullet}(L, \mathbf{R})$ is isomorphic to the cohomology of the complex of $L$-invariant differential forms on $X$.

\section{Bounded Cohomology and Bounded Differential Forms}

3.1. Bounded Cohomology. Let $G$ be a locally compact second countable group. The continuous bounded cohomology $\mathrm{H}_{\mathrm{cb}}^{\bullet}(G, \mathbf{R})$ of $G$ is the cohomology of the complex of $G$-invariants

$$
0 \longrightarrow C_{\mathrm{b}}(G, \mathbf{R})^{G \stackrel{d}{\longrightarrow}} C_{\mathrm{b}}\left(G^{2}, \mathbf{R}\right)^{G} \stackrel{d}{\longrightarrow} \cdots
$$


where

$$
\begin{aligned}
C_{\mathrm{b}}\left(G^{k+1}, \mathbf{R}\right):=\left\{f: G^{k+1} \rightarrow \mathbf{R} \mid f\right. \text { is continuous and } \\
\left.\qquad\|f\|_{\infty}=\sup _{\left(g_{0}, \ldots, g_{k}\right) \in G^{k+1}}\left|f\left(g_{0}, \ldots, g_{k}\right)\right|<\infty\right\}
\end{aligned}
$$

is a $G$-module via

$$
(h f)\left(g_{0}, \ldots, g_{k}\right)=f\left(h^{-1} g_{0}, \ldots, h^{-1} g_{k}\right) .
$$

and $d$ is the homogeneous coboundary operator

$$
d f\left(g_{0}, \ldots g_{k+1}\right):=\sum_{i=0}^{k+1} f\left(g_{0}, \ldots, \hat{g}_{i}, \ldots, g_{k+1}\right) .
$$

The inclusion of complexes $C_{\mathrm{b}}\left(G^{\bullet+1}, \mathbf{R}\right) \subset C\left(G^{\bullet+1}, \mathbf{R}\right)$, where $C\left(G^{\bullet+1}, \mathbf{R}\right)$ denotes the space of continuous real valued functions induces a natural comparison map

$$
c_{G}^{\bullet}: \mathrm{H}_{\mathrm{cb}}^{\bullet}(G, \mathbf{R}) \rightarrow \mathrm{H}_{\mathrm{c}}^{\bullet}(G, \mathbf{R})
$$

from continuous bounded cohomology to continuous cohomology.

The comparison map is in general neither injective nor surjective and its behavior is not well understood except in very special cases.

3.2. Factoring Through Bounded Differential Forms. For the case that $G$ is a connected semisimple Lie group (of noncompact type) with finite center, $X$ the associated symmetric space and $L<G$ a closed subgroup, Burger and Iozzi showed in [3] that there is a map $\delta_{\infty}^{\bullet}$ such that the following diagram commutes

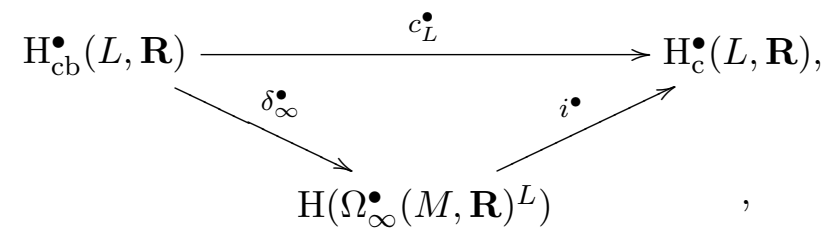

where $i^{\bullet}$ is the natural map induced by the inclusion $\Omega_{\infty}(X, \mathbf{R})^{L} \subset \Omega(X, \mathbf{R})^{L}$.

Let us describe the construction of the map $\delta_{\infty}^{\bullet}$ in more detail. It relies on the functorial approach to continuous bounded cohomology (see $[19,7]$ ) in particular on the fact that the continuous bounded cohomology of $L$ can be realized by bounded measurable functions on $(G / P)^{\bullet}$, where $P<G$ is a minimal parabolic subgroup. 
Let $\nu_{0}$ be the unique $K$-invariant probability measure on $G / P$ and denote by

$$
L_{\text {alt }}^{\infty}\left((G / P)^{k+1}, \nu_{0}^{k+1}\right)
$$

the space of alternating bounded measurable functions on $(G / P)^{k+1}$, equipped with the structure of an $L$-module as above. Then $\mathrm{H}_{\mathrm{cb}}^{\bullet}(L, \mathbf{R})$ is canonically isometrically isomorphic to the cohomology of the complex of $L$-invariants

$$
0 \longrightarrow L_{\text {alt }}^{\infty}\left((G / P), \nu_{0}\right)^{L} \stackrel{d}{\longrightarrow} L_{\text {alt }}^{\infty}\left((G / P)^{2}, \nu_{0}^{2}\right)^{L} \stackrel{d}{\longrightarrow} \cdots
$$

where $d$ is the homogeneous boundary operator.

The homogeneous space $G / P$ can be embedded into the visual boundary $\partial X$. For this let $\mathfrak{a}^{+} \subset \mathfrak{a}$ be the positive Weyl chamber associated to a choice of positive roots $\Psi^{+}$of $\mathfrak{g}$ relative to $\mathfrak{a}$. Let $b \in \mathfrak{a}^{+}$be the barycenter, that is the vector predual to the sum of all positive roots in $\Psi^{+}$. Let $\xi_{b} \in \partial X$ be the point represented by the geodesic ray starting at $x_{0}$ in the direction of $b$. Then $\xi_{b}$ is regular and its $G$-orbit $G \xi_{b} \subset \partial X$ is naturally identified with $G / P$. The measure $\nu_{0}$ on $G / P$ defines a probability measure $\nu_{0}$ on $\partial X$ with support on $G / P \subset \partial X$.

For any $\xi \in \partial X$ let us define a function

$$
\begin{aligned}
e^{\xi}: X & \rightarrow \mathbf{R} \\
x & \mapsto e^{-h(X) B_{\xi}^{0}(x)},
\end{aligned}
$$

where $h(X)$ is the volume entropy of $X$.

Proposition 3.1. [3, Lemma 3.3.] Let $c \in L_{\text {alt }}^{\infty}\left((\partial X)^{k+1}, \nu_{0}^{k+1}\right)$. Then

$$
\alpha_{c}:=\int_{(\partial X)^{k+1}}\left(c\left(\xi_{0}, \cdots, \xi_{k}\right) e^{\xi_{0}} d e^{\xi_{1}} \wedge \cdots \wedge d e^{\xi_{k}}\right) d \nu_{0}\left(\xi_{0}, \cdots, \xi_{k}\right)
$$

is a bounded differential form. Moreover, the map

$$
\begin{aligned}
\delta_{\infty}^{\bullet}: L_{\text {alt }}^{\infty}\left((\partial X)^{\bullet+1}, \nu_{0}^{\bullet+1}\right) & \rightarrow \Omega_{\infty}^{\bullet}(X) \\
c & \mapsto \alpha_{c}
\end{aligned}
$$

is a G-invariant map of complexes.

Remark 3.2. Note that even in the case when $\kappa \in \mathrm{H}_{\mathrm{c}}^{k}(L, \mathbf{R})$ is known to be represented by a bounded cocycle, this construction gives a very specific representative for $\kappa$. For applications we refer the reader to [3]. 
Corollary 3.3. Let $\kappa \in \mathrm{H}_{\mathrm{c}}^{k}(L, \mathbf{R})$. Assume that $\kappa$ lies in the image of $c_{L}^{(k)}$, then $\kappa$ can be represented by a bounded L-invariant differential form $\alpha$ on $M$ which admits a bounded primitive $\beta \in \Omega_{\infty}^{k-1}(M, \mathbf{R})$.

Proof. Assume that $\kappa=c_{L}^{(k)}\left(\kappa_{\mathrm{b}}\right)$ and let $c_{\kappa} \in L_{\text {alt }}^{\infty}\left((\partial X)^{k+1}, \nu_{0}^{k+1}\right)^{L}$ be a cocycle representing $\kappa_{\mathrm{b}}$. The complex

$$
0 \longrightarrow L_{\text {alt }}^{\infty}\left((G / P), \nu_{0}\right) \stackrel{d}{\longrightarrow} L_{\text {alt }}^{\infty}\left((G / P)^{2}, \nu_{0}^{2}\right) \stackrel{d}{\longrightarrow} \cdots
$$

is exact and so there exists $b_{\kappa} \in L_{\text {alt }}^{\infty}\left((\partial X)^{k}, \nu_{0}^{k}\right)$ with $d b_{\kappa}=c_{\kappa}$. Therefore setting $\alpha=\delta_{\infty}^{(k+1)}\left(c_{\kappa}\right)$ and $\beta=\delta_{\infty}^{(k)}\left(b_{\kappa}\right)$ Proposition 3.1 implies that $\alpha$ is a bounded $L$-invariant $k$-form representing $\kappa$ and $\beta$ is a bounded $(k-1)$-form satisfying $d \beta=\alpha$.

So Proposition 3.1 does give necessary conditions for cohomology classes to be represented by bounded classes, which by Corollary 2.2 and Corollary 2.4 are satisfied for example

(i) if $L<G$ is a cocompact lattice and the degree $k \geq r_{X}+1$, or

(ii) if $L=G$.

\section{Gersten's $l_{\infty}$-COHOMOlOGY}

When $\Gamma$ is a finitely generated group and $X$ a $K(\Gamma, 1)$-space with finite $n$ skeleton Gersten defined the $l_{\infty}$-cohomology (or also bounded valued cohomology) $\mathrm{H}_{\infty}^{i}(\Gamma, \mathbf{R})$ for $i \leq n$ (see [10]).

Let us denote an $i$-cell of $X$ by $e^{(i)}$. The $l_{1}$-norm $\|\cdot\|_{1}$ of a chain $c$ is the sum of the absolute values of the coefficients of $c$ with respect to a natural basis of cells of $X$.

Definition 4.1. A $C W$-complex $U$ is said to have bounded geometry in dimension $\leq n$ if there exists some $M \in \mathbf{N}$ such that $\left\|\partial e^{(i)}\right\|_{1} \leq M$ for all $i$-cells $e^{(i)}$ of $U$ and all $i \leq n$.

Let $\widetilde{X}$ be the universal cover of $X$ and $\pi: \widetilde{X} \rightarrow X$ the covering map. Then $\widetilde{X}$ is carries a $C W$-structure induced from $X, \tilde{e}^{(i)}=\pi^{*} e^{(i)}$, with respect to which it has bounded geometry in dimension $\leq n$. 
For all $i \leq n$ define

$$
C_{\infty}^{i}(\tilde{X}, \mathbf{R}) \subset C^{i}(\widetilde{X}, \mathbf{R})
$$

to be the subset of bounded valued cochains, that is

$$
C_{\infty}^{i}(\widetilde{X}, \mathbf{R}):=\left\{\phi \in C^{i}(\widetilde{X}, \mathbf{R}) \mid \exists N \in \mathbf{N} \text { such that }\left|\phi\left(\tilde{e}^{(i)}\right)\right| \leq N\right\} .
$$

Since $\widetilde{X}$ has bounded geometry the co-boundary

$$
\delta: C^{i}(\widetilde{X}, \mathbf{R}) \rightarrow C^{i+1}(\tilde{X}, \mathbf{R})
$$

satisfies $\delta\left(C_{\infty}^{i}(\tilde{X}, \mathbf{R})\right) \subset C_{\infty}^{i+1}(\tilde{X}, \mathbf{R})$ for all $i \leq n$.

The $l_{\infty}$-cohomology (or bounded valued cohomology) is defined to be the cohomology of this subcomplex:

$$
\mathrm{H}_{\infty}^{i}(\Gamma, \mathbf{R}):=\mathrm{H}_{\infty}^{i}(\tilde{X}, \mathbf{R})
$$

Gersten proved that $\mathrm{H}_{\infty}^{i}(\Gamma, \mathbf{R})$ indeed does not depend on the choice of a $K(\Gamma, 1)$ space with finite $n$-skeleton. Moreover he proves [9, Theorem 11.4.] that if $\Gamma, \Gamma^{\prime}$ are quasi-isometric and both groups admit an Eilenberg-MacLane space with finite $n$-skeleton, then for all $i \leq n$

$$
\mathrm{H}_{\infty}^{i}(\Gamma, \mathbf{R}) \cong \mathrm{H}_{\infty}^{i}\left(\Gamma^{\prime}, \mathbf{R}\right)
$$

Remark 4.2. A finitely presented group $\Gamma$ always admits a $K(\Gamma, 1)$-space with finite 2-skeleton, so that $\mathrm{H}_{\infty}^{2}(\Gamma, \mathbf{R})$ is well defined. It is strongly related to isoperimetric inequalities in $\Gamma$.

There is a natural map $h_{\Gamma}: \mathrm{H}^{i}(\Gamma, \mathbf{R}) \rightarrow \mathrm{H}_{\infty}^{i}(\Gamma, \mathbf{R})$ constructed as follows: Since $X$ is a $K(\Gamma, 1)$-space we have that $\mathrm{H}^{i}(\Gamma, \mathbf{R})=\mathrm{H}^{i}(X, \mathbf{R})$. Let $f \in C^{i}(X, \mathbf{R})$ be a cochain and $\pi^{*} f \in C^{i}(\widetilde{X}, \mathbf{R})$ its lift. Then

$$
\sup _{\tilde{e}^{(i)}}\left\|\pi^{*} f\left(\tilde{e}^{(i)}\right)\right\|=\sup _{e^{(i)}}\left\|f\left(e^{(i)}\right)\right\|,
$$

which is obviously bounded for $i \leq n$ since $X$ has a finite $n$-skeleton.

Lemma 4.3. [9, Proposition 10.2.] The composition of natural maps

$$
\mathrm{H}_{\mathrm{b}}^{i}(\Gamma, \mathbf{R}) \stackrel{c_{\Gamma}}{\longrightarrow} \mathrm{H}^{i}(\Gamma, \mathbf{R}) \stackrel{h_{\Gamma}}{\longrightarrow} \mathrm{H}_{\infty}^{i}(\Gamma, \mathbf{R})
$$

is the zero map 
We will not recall Gersten's proof here, because it is immediate once we consider our variant of bounded valued cohomology in the next section.

In the special situation when $X$ is an aspherical manifold with fundamental group $\pi_{1}(X)=\Gamma$, Gersten's $l_{\infty}$-cohomology is related to the bounded deRham cohomology of $\widetilde{X}$.

Proposition 4.4. [11, Proposition 12.2] The $l_{\infty}$-cohomology $\mathrm{H}_{\infty}^{i}(\Gamma, \mathbf{R})=$ $\mathrm{H}_{\infty}^{i}(\tilde{X}, \mathbf{R})$ is isomorphic to a direct summand of $\mathrm{H}_{\mathrm{bdR}}^{i}(\tilde{X}, \mathbf{R})$.

The proof of this goes back to a construction of Whitney [21, page 139].

Corollary 2.2 implies

Corollary 4.5. Let $G$ be a connected semisimple Lie group with finite center, $M$ its associated symmetric space, and $\Gamma<G$ a cocompact lattice, then

$$
h_{\Gamma}^{\bullet}: \mathrm{H}(\Gamma, \mathbf{R}) \rightarrow \mathrm{H}_{\infty}(\Gamma, \mathbf{R})
$$

is the zero map in degrees $\bullet \geq r_{M}+1$.

\section{A Variant of Bounded Valued Cohomology}

Let $G$ be a locally compact second countable group. The space $C_{\mathrm{b}}(G, \mathbf{R})$ of bounded continuous functions on $G$ is a Banach $G$-module with respect to the supremum-norm and the $G$-action $(h f)(g):=f\left(h^{-1} g\right)$ for all $h, g \in G$.

In particular we can consider continuous cohomology of $G$ with coefficients in $C_{\mathrm{b}}(G, \mathbf{R})$. That is what we want to call (continuous) bounded valued cohomology of $G$ (with coefficients in $\mathbf{R}$ ):

$$
\mathrm{H}_{\mathrm{c}, \infty}^{i}(G, \mathbf{R}):=\mathrm{H}_{\mathrm{c}}^{i}\left(G, C_{\mathrm{b}}(G, \mathbf{R})\right) .
$$

The inclusion of coefficients $\mathbf{R} \rightarrow C_{\mathrm{b}}(G, \mathbf{R})$, where $\mathbf{R}$ is identified with the constant functions, gives rise to a natural map

$$
h_{G}^{\bullet}: \mathrm{H}_{\mathrm{c}}^{\bullet}(G, \mathbf{R}) \rightarrow \mathrm{H}_{\mathrm{c}, \infty}^{\bullet}(G, \mathbf{R}) .
$$

Proposition 5.1. The composition of natural maps

$$
\mathrm{H}_{\mathrm{cb}}^{\bullet}(G, \mathbf{R}) \stackrel{c_{G}^{\bullet}}{\longrightarrow} \mathrm{H}_{\mathrm{c}}^{\bullet}(G, \mathbf{R}) \stackrel{h_{G}^{\bullet}}{\longrightarrow} \mathrm{H}_{\mathrm{c}, \infty}^{\bullet}(G, \mathbf{R})
$$

is zero in positive degrees. 
Proof. Consider the commutative diagram

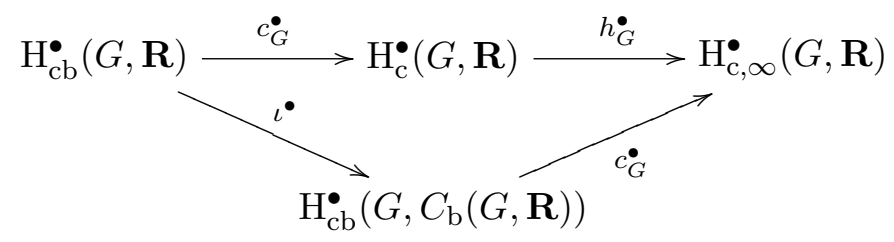

and note that $\iota^{\bullet}$ is the zero map in positive degrees, because $C_{\mathrm{b}}(G, \mathbf{R})$ is a relative injective coefficient module (see [19, Proposition 4.14 and Proposition 7.4.1]).

Proposition 5.2. Let $\Gamma$ be a finitely generated group and $X$ a $K(\Gamma, 1)$-space with finite $n$-skeleton. Then

$$
\mathrm{H}_{\infty}^{i}(\Gamma, \mathbf{R}) \cong \mathrm{H}_{\mathrm{c}, \infty}^{i}(\Gamma, \mathbf{R})
$$

for all $i \leq n$.

Proof. Since $X$ is a classifying space for $\Gamma$ we have that $\mathrm{H}_{\mathrm{c}, \infty}^{i}(\Gamma, \mathbf{R})=$ $\mathrm{H}^{i}\left(X, C_{\mathrm{b}}(G, \mathbf{R})\right)$. Let $\widetilde{X}$ be the universal cover of $X$, endowed with the induced CW-structure. For every $i$-cell $e^{(i)}$ of $X$ let us choose coherently (in a $\Gamma$-equivariant way) a lift $\tilde{e}_{0}^{(i)}$, which is an $i$-cell of $\tilde{X}$, so that we enumerate all $i$-cells $\tilde{e}^{(i)}$ of $\tilde{X}$ by elements $\gamma \in \Gamma$, where $\tilde{e}_{\gamma}^{(i)}=\gamma\left(\tilde{e}_{0}^{(i)}\right)$. We define

$$
\begin{aligned}
\Phi: C^{i}\left(X, C_{\mathrm{b}}(G, \mathbf{R})\right) & \rightarrow C_{\infty}^{i}(\tilde{X}, \mathbf{R}) \\
f & \mapsto \Phi(f),
\end{aligned}
$$

where $\Phi(f)\left(\tilde{e}_{\gamma}^{(i)}\right):=f\left(e^{(i)}\right)\left(\gamma^{-1}\right)$, and

$$
\begin{aligned}
\Psi: C_{\infty}^{i}(\widetilde{X}, \mathbf{R}) & \rightarrow C^{i}\left(X, C_{\mathrm{b}}(G, \mathbf{R})\right) \\
\alpha & \mapsto \Psi(\alpha),
\end{aligned}
$$

where $\Psi(\alpha)\left(e^{(i)}\right)(\gamma):=\alpha\left(\tilde{e}_{\gamma^{-1}}^{(i)}\right)$. Then clearly $\Phi$ and $\Psi$ commute with the differentials. Moreover we have

$$
\Psi \circ \Phi(f)\left(e^{(i)}\right)(\gamma)=\Phi(f)\left(\tilde{e}_{\gamma^{-1}}^{(i)}\right)=f\left(e^{(i)}\right)(\gamma)
$$

and

$$
\Phi \circ \Psi(\alpha)\left(\tilde{e}_{\gamma}^{(i)}\right)=\Psi(\alpha)\left(e^{(i)}\right)(\gamma)=\alpha\left(\tilde{e}_{\gamma}^{(i)}\right) .
$$

So $\Phi$ and $\Psi$ induce isomorphisms on the level of cohomology.

The following Proposition was proved by Gersten [9, Theorem 10.13] for $l_{\infty^{-}}$ cohomology of finitely generated groups. 
Proposition 5.3. Let $G$ be amenable, then

$$
h_{G}^{\bullet}: \mathrm{H}_{\mathrm{c}}^{\bullet}(G, \mathbf{R}) \rightarrow \mathrm{H}_{\mathrm{c}, \infty}^{\bullet}(G, \mathbf{R}) .
$$

is injective.

Proof. If $G$ is amenable the inclusion of coefficients

$$
\mathbf{R} \rightarrow C_{\mathrm{b}}(G, \mathbf{R})
$$

has a norm one left inverse $G$-morphism $C_{\mathrm{b}}(G, \mathbf{R}) \rightarrow \mathbf{R}$ (see for example [19, Corollary 5.1.3.]), which yields a left inverse to $h_{G}^{\bullet}$.

Finally we would like to relate $\mathrm{H}_{\mathrm{c}, \infty}^{\bullet}(G, \mathbf{R})$ to the bounded deRham cohomology of some associated space $X$. In general this will not be possible. However in the situation that $G$ is a connected semisimple Lie group with finite center and $X$ is the associated symmetric space we have:

Proposition 5.4. Let $L<G$ be a closed subgroup. Assume that $L$ acts transitively on $X$. Then the cohomology $\mathrm{H}_{\mathbf{c}, \infty}^{\bullet}(L, \mathbf{R})$ is isomorphic to a direct summand of $\mathrm{H}^{\bullet}\left(\Omega_{\infty}(X)\right)$

Proof. Note first that $\mathrm{H}_{\mathrm{c}, \infty}^{\bullet}(L, \mathbf{R}) \cong \Omega^{\bullet}\left(X, C_{b}(L, \mathbf{R})\right)^{L}$. The action of $L$ on $\Omega^{\bullet}\left(X, C_{b}(L, \mathbf{R})\right)$ is given by

$$
\left(g^{*} \alpha\right)_{p}\left(v_{1}, \ldots, v_{k}\right)(h)=\alpha_{g^{-1} p}\left(g_{*} v_{1}, \ldots g_{*} v_{k}\right)\left(g^{-1} h\right) .
$$

Let us define

$$
\begin{aligned}
\Phi: \Omega^{k}\left(X, C_{b}(L, \mathbf{R})\right)^{L} & \rightarrow \Omega_{\infty}^{k}(X, \mathbf{R}) \\
\alpha & \mapsto \Phi(\alpha),
\end{aligned}
$$

where $\Phi(\alpha)_{p}\left(v_{1}, \ldots, v_{k}\right):=\alpha_{p}\left(v_{1}, \ldots v_{k}\right)(1)$. This map $\Phi$ is well defined. To see this let us fix a base point $x_{0} \in X$. Then $\sup _{g \in L} \sup _{v_{1}, \ldots, v_{k} \in T_{x_{0}}^{1} X}\left|\alpha_{x_{0}}\left(v_{1}, \ldots, v_{k}\right)(g)\right|$ is bounded by a constant $K_{x_{0}}$. Since $L$ acts transitively on $X$ by isometries, we can choose $g_{p} \in L$ such that $g_{p}\left(x_{0}\right)=p$. Then

$$
\begin{aligned}
\left|\alpha_{p}\left(v_{1}, \ldots, v_{k}\right)(1)\right| & =\left|\left(g_{p}^{*} \alpha\right)_{p}\left(v_{1}, \ldots, v_{k}\right)(1)\right| \\
& =\left|\alpha_{g^{-1}}\left(g_{*} v_{1}, \ldots g_{*} v_{k}\right)\left(g^{-1}\right)\right| \\
& =\left|\alpha_{x_{0}}\left(g_{*} v_{1}, \ldots g_{*} v_{k}\right)\left(g^{-1}\right)\right| \leq K_{x_{0}}
\end{aligned}
$$


hence $\Phi(\alpha) \in \Omega_{\infty}^{k}(X, \mathbf{R})$. Define

$$
\begin{aligned}
\Psi: \Omega_{\infty}^{k}(X, \mathbf{R}) & \rightarrow \Omega^{k}\left(X, C_{b}(L, \mathbf{R})\right)^{L} \\
\beta & \mapsto \Psi(\beta),
\end{aligned}
$$

where $\Psi(\beta)_{p}\left(v_{1}, \ldots, v_{k}\right)(g):=\left(g^{*} \beta\right)_{p}\left(v_{1}, \ldots, v_{k}\right)$. Then $\Psi$ and $\Phi$ commute with the differentials and we have

$$
\begin{aligned}
\Psi \circ \Phi(\alpha)_{p}\left(v_{1}, \ldots, v_{k}\right)(g) & =\Phi(\alpha)_{g^{-1} p}\left(g_{*} v_{1}, \ldots, g_{*} v_{k}\right) \\
& =\alpha_{g^{-1} p}\left(g_{*} v_{1}, \ldots, g_{*} v_{k}\right)(1) \\
& =\left(g^{*} \alpha\right)_{p}\left(v_{1}, \ldots, v_{k}\right)(g)=\alpha_{p}\left(v_{1}, \ldots, v_{k}\right)(g),
\end{aligned}
$$

where the last equality holds because $\alpha$ is $L$-invariant.

This proposition together with Corollary 2.4 implies

Corollary 5.5. The natural map $\mathrm{H}_{G}^{\bullet}: \mathrm{H}_{\mathrm{c}}^{\bullet}(G, \mathbf{R}) \rightarrow \mathrm{H}_{\mathrm{c}, \infty}^{\bullet}(G, \mathbf{R})$ is zero.

Bounded valued cohomology can be defined for more general coefficient modules $E$ (instead of the trivial coefficients) as defined in [19]. Suitable adaptations of Proposition 5.2 and Proposition 5.3 hold in this context.

\section{REFERENCES}

1. M. Bucher-Karlsson, Simplicial volume of locally symmetric spaces covered by $S L(3, R) / S O(3)$, Geom. Dedicata 125 (2007), no. 1, 203-224.

2. M. Burger and A. Iozzi, Bounded Kähler class rigidity of actions on Hermitian symmetric spaces, Ann. Sci. École Norm. Sup. (4) 37 (2004), no. 1, 77-103.

3. __ Bounded differential forms, generalized Milnor-Wood inequality and applications to rigidity theory, Geom. Dedicata 125 (2007), no. 1, 1-23.

4. M. Burger, A. Iozzi, and A. Wienhard, Hermitian symmetric spaces and Kähler rigidity, Transformation Groups 12 (2007), no. 1, 5-32.

5. __ Representations of surface groups with maximal Toledo invariant, Annals of Mathematics 172 (2010), no. 1, 517-566.

6. - Tight embeddings, GAFA 19 (2009), no. 3, 678-721.

7. M. Burger and N. Monod, Continuous bounded cohomology and applications to rigidity theory, Geom. Funct. Anal. 12 (2002), no. 2, 219-280. MR MR1911660 (2003d:53065a)

8. K. Corlette, L. Hernández Lamoneda, and A. Iozzi, A vanishing theorem for the tangential de Rham cohomology of a foliation with amenable fundamental groupoid, Geom. Dedicata 103 (2004), 205-223.

9. S. M. Gersten, Bounded cohomology and combings of groups, Preprint, version 5.5.

10. Cohomological lower bounds for isoperimetric functions on groups, Preprint 1995. 
11. A note on cohomological vanishing and the linear isoperimetric inequality, Preprint 1996.

12. M. Gromov, Hyperbolic manifolds, groups and actions, Riemann surfaces and related topics: Proceedings of the 1978 Stony Brook Conference (State Univ. New York, Stony Brook, N.Y., 1978) (Princeton, N.J.), Ann. of Math. Stud., vol. 97, Princeton Univ. Press, 1981, pp. $183-213$.

13. __ Kähler hyperbolicity and $L_{2}$-Hodge theory, J. Differential Geom. 33 (1991), no. 1, 263-292.

14. __ Asymptotic invariants of infinite groups, Geometric group theory, Vol. 2 (Sussex, 1991), London Math. Soc. Lecture Note Ser., vol. 182, Cambridge Univ. Press, Cambridge, 1993, pp. 1-295.

15. J. Kedra, Symplectically hyperbolic manifolds, Differential Geom. Appl. 27 (2009), no. 4, 455-463.

16. J.-F. Lafont and B. Schmidt, Simplicial volume of closed locally symmetric spaces of noncompact type, Acta Math. 197 (2006), no. 1, 129-143.

17. C. T. McMullen, The moduli space of Riemann surfaces is Kähler hyperbolic, Ann. of Math. (2) 151 (2000), no. 1, 327-357.

18. I. Mineyev, Straightening and bounded cohomology of hyperbolic groups, Geom. Funct. Anal. 11 (2001), no. 4, 807-839.

19. N. Monod, Continuous bounded cohomology of locally compact groups, Lecture Notes in Mathematics, vol. 1758, Springer-Verlag, Berlin, 2001.

20. Leonid Polterovich, Growth of maps, distortion in groups and symplectic geometry, Invent. Math. 150 (2002), no. 3, 655-686.

21. H. Whitney, Geometric integration theory, Princeton University Press, Princeton, N. J., 1957.

Anna Wienhard

Department of Mathematics

Princeton University

Fine Hall, Washington Road

Princeton, NJ 08540, USA

E-mail: wienhard@math.princeton.edu 